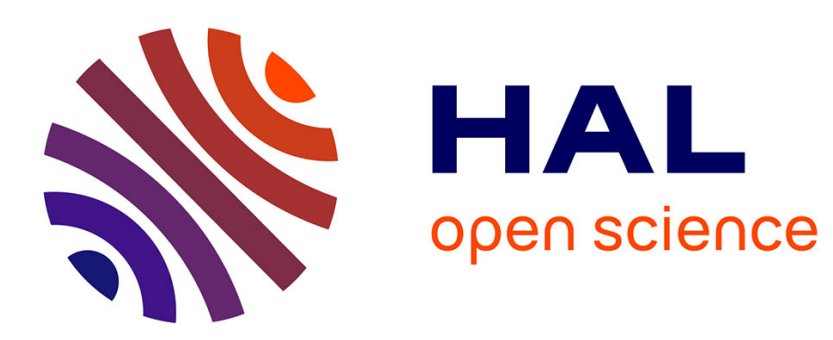

\title{
Un cryostat pour la spectroscopie optique et radiofréquence en champ fort
}

\author{
R. Adde, J. Pontnau
}

\section{To cite this version:}

R. Adde, J. Pontnau. Un cryostat pour la spectroscopie optique et radiofréquence en champ fort. Revue de Physique Appliquée, 1970, 5 (6), pp.811-812. 10.1051/rphysap:0197000506081100 . jpa00243458

\section{HAL Id: jpa-00243458 https://hal.science/jpa-00243458}

Submitted on 1 Jan 1970

HAL is a multi-disciplinary open access archive for the deposit and dissemination of scientific research documents, whether they are published or not. The documents may come from teaching and research institutions in France or abroad, or from public or private research centers.
L'archive ouverte pluridisciplinaire HAL, est destinée au dépôt et à la diffusion de documents scientifiques de niveau recherche, publiés ou non, émanant des établissements d'enseignement et de recherche français ou étrangers, des laboratoires publics ou privés. 


\title{
UN CRYOSTAT POUR LA SPECTROSCOPIE OPTIQUE ET RADIOFRÉQUENCE EN CHAMP FORT
}

\author{
par R. ADDE et J. PONTNAU \\ Institut d'Electronique Fondamentale, Laboratoire associé au C. N. R. S., \\ Bâtiment 220, Faculté des Sciences, 91, Orsay
}

(Reçu le 19 juin 1970)

\begin{abstract}
Résumé. - Dans cet appareillage, le champ magnétique $(50 \mathrm{kG})$ est produit par des bobines supraconductrices placées en position d'Helmholtz dans un cryostat indépendant du cryostat optique contenant l'échantillon. Ainsi, la température de l'échantillon est réglable indépendamment de celle de la bobine. Des fenêtres permettent l'accès optique transversal et axial au centre du système.
\end{abstract}

Abstract. - In this device, the magnetic field $(50 \mathrm{kG})$ is obtained with supraconductive Helmholtz coils in a cryostat independant of the sample cryostat. In this way, the sample temperature may be controled independantly of the coil temperature. Optical windows allow transversal and axial optical access to the center of the system.

Les dispositifs de spectroscopie optique et radiofréquence en champ fort sont le plus souvent constitués par un solénoïde supraconducteur et un porte-échantillon placés dans le même Dewar. Ce système est peu pratique car il rend difficile le contrôle et le réglage de la température de l'échantillon indépendamment de celle de la bobine. De plus la configuration géométrique de ces systèmes allonge considérablement le trajet optique, nécessite l'emploi de miroirs qui rendent difficile l'alignement optique et introduisent des effets de dépolarisation. Il en résulte des pertes importantes de sensibilité.

Nous décrivons ici un appareillage (Fig. 1) qui résout ces difficultés : le champ magnétique $(50 \mathrm{kG})$ est produit par des bobines d'Helmholtz supraconductrices $(1,2)$ placées dans un cryostat (I) indépendant du cryostat contenant l'échantillon (II). Le cryostat (I) possède des fenêtres transparentes (3) permettant l'accès optique transversal au centre du système. Il présente en outre un accès cylindrique axial de $\varnothing 52 \mathrm{~mm}$ à la température ordinaire qui reçoit le doigt non argenté du cryostat optique (II) ; les dimensions du doigt permettent de placer l'échantillon dans la cavité rectangulaire d'un spectrographe en bande $X$.

1. Bobine supraconductrice. -- Deux solénoödes identiques placés en position d'Helmholtz composent la bobine supraconductrice. Le champ magnétique axial au voisinage du centre du système se développe suivant les puissances paires croissantes de la coordonnée axiale, le terme d'ordre 2 est annulé par un choix judicieux des dimensions géométriques, le terme d'ordre 4 est le premier terme non nul. Les calculs ont été effectués en utilisant les tables de Girard et Sauzade [1]. Nous avons choisi d'employer du fil supra-

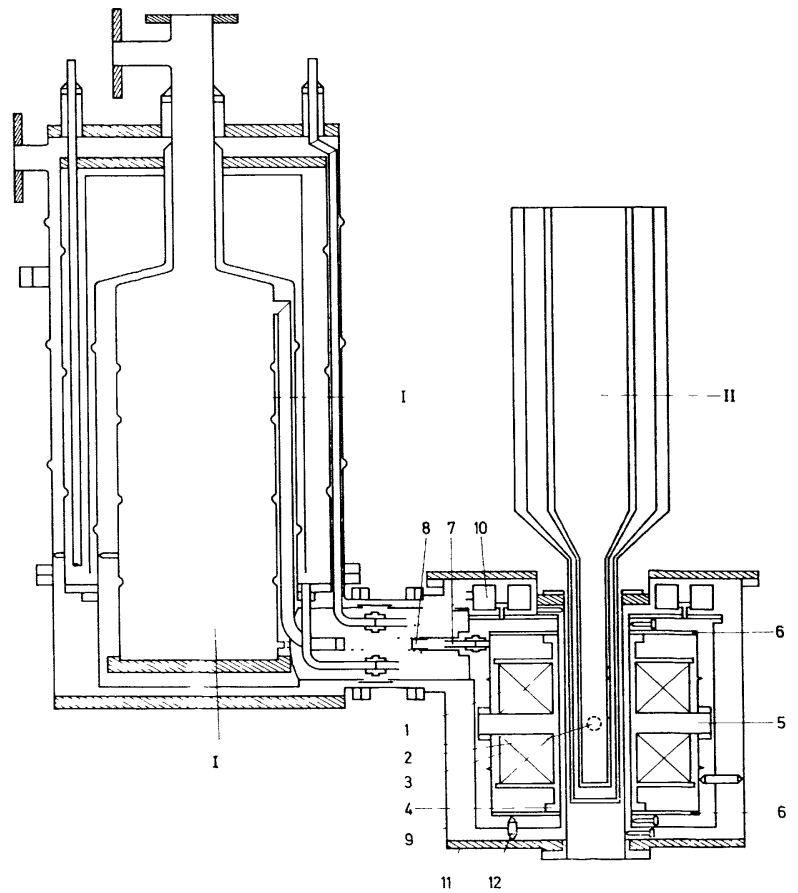

Fig. 1.

conducteur de petite section car il permet : a) la construction de deux bobinages aussi identiques que possible pour atteindre l'homogénie théorique, $b$ ) des câbles d'amenée de courant de petite section, condition imposée par la géométrie du cryostat ; $c$ ) une alimentation à courant relativement faible ; $d$ ) une bobine de grande self-inductance; cette dernière propriété permet d'obtenir un balayage très linéaire lorsque l'alimentation maintient une tension constante aux bornes. Notre choix s'est fixé sur du fil de $\mathrm{Nb}-40 \% \mathrm{Ti}$ de 
$0,25 \mathrm{~mm}$ recouvert d'une couche de $70 \mu$ de cuivre pour le stabiliser (fil supraconducteur Thomson-CSF type THN 91025071 ). Chaque bobinage contient 13650 spires et a pour dimensions $\Phi_{i}=76,2 \mathrm{~mm}$, $\Phi_{e}=190 \mathrm{~mm}, l=48 \mathrm{~mm}$. La distance entre leurs axes transversaux est de $68 \mathrm{~mm}$. La réalisation des bobinages a été très soignée afin que les enroulements soient symétriques par rapport au centre du système. L'isolation de couches pour éviter les claquages au moment des transitions est réalisée avec un ruban de mylar de $30 \mu$ d'épaisseur. Le champ au centre est de $1800 \mathrm{G} / \mathrm{A}$. Nous obtenons couramment $50 \mathrm{kG}$ et avons atteint $57 \mathrm{kG}$ au moment de la transition. L'inhomogénie est de $10^{-4}$ dans un volume de $1 \mathrm{~cm}^{3}$.

2. Cryostat de la bobine supraconductrice. - Pour supporter les forces axiales, le mandrin (4) des deux bobinages est réalisé d'une seule pièce ; ils sont séparés par une flasque massive (5) percée radialement d'un trou de $\varnothing 16 \mathrm{~mm}$ pour l'accès transversal au centre dela bobine. De part et d'autre de la flasque centrale, deux capots (6) s'emboîtent sur le mandrin, l'ensemble constituant l'enceinte à hélium du bobinage ; l'étanchéité est assurée par des joints en indium. Le passage de l'hélium de part et d'autre de la flasque centrale s'effectue par une série de trous percés axialement à la périphérie des bobinages. L'enceinte a une contenance de 0,51 d'hélium; des emplacements sont prévus pour les contacts supraconducteurs. L'enceinte à hélium de la bobine et le vase à hélium du cryostat d'alimentation sont reliés par deux canalisations, l'une pour l'arrivée d'hélium liquide (7) l'autre pour l'évacuation de l'hélium gazeux (8); des raccords Winkler assurent un montage aisé.

L'enceinte à hélium est placée à l'intérieur d'une chemise en laiton (9) maintenue à $77^{\circ} \mathrm{K}$ par contact thermique avec un vase de 0,5 1 d'azote liquide (10) fixé sur son sommet; elle est alimentée à partir du vase à azote du cryostat principal par des canalisations munies de raccords Winkler. L'enceinte extérieure (11) laisse un volume accessible à température ordinaire de $\varnothing=52 \mathrm{~mm}$ dans l'axe de la bobine ; des fenêtres en quartz permettent l'accès optique transversal. Des pieds coniques en permaglass (12) assurent l'isolement thermique entre l'enceinte extérieure, l'enceinte à $77^{\circ}$ et la bobine supraconductrice.

Le cryostat d'alimentation (I) est démontable par le fond pour faciliter le montage des connexions de la bobine supraconductrice (câbles d'amenée de courant, prises de potentiel) et celles des jauges de niveau d'hélium ; il est de conception classique comme le cryostat optique. La consommation de 1,2 1 d'hélium par heure est très raisonnable étant donné la complexité du système et donne une autonomie de fonctionnement de six heures, largement suffisante pour ne pas perturber la plupart des expériences.

\section{Bibliographie}

[1] Girard (B.) et Sauzade (M.), Nucl. Inst. Methods, 1964, 25, 269. 